\title{
molecules
}

ISSN 1420-3049

(C) 2007 by MDPI

www.mdpi.org/molecules

\author{
Full Paper
}

\section{Novel and Efficient Synthesis of $N, N$-Dialkylamino- and $O$-alkylphenyl-2-(1-alkyl/phenyl-2-oxopropylidene) Phosphonohydrazido Oximes - Potential Marine Fish Toxin Analogues. Part 2}

\section{Rajesh Kumar, Arvind K. Gupta and Mahabir P. Kaushik*}

Process Technology Development Division, Defence R \& D Establishment, Jhansi Road, Gwalior474002 (MP), India; Tel. (+91) 751-2343972; Fax (+91) 751-2341148

* Author to whom correspondence should be addressed; e-mail: mpkaushik@rediffmail.com. For Part 1 of this series, see reference 13a.

Received: 6 July 2007; in revised form: 26 July 2007 / Accepted: 26 July 2007 / Published: 2 August 2007

\begin{abstract}
A novel and efficient method for the synthesis of $N, N$-dialkylamino/O-alkyl phenyl-2-(1-alkyl/phenyl-2-oxopropylidene) phosphonohydrazido oximes $\mathbf{4}$ using activated silica has been developed. The reaction involves the condensation of substituted $\alpha$ ketooxime and $\mathrm{N}, \mathrm{N}$-dialkylaminophenylphosphonohydrazides or $\mathrm{O}$-alkylphenylphosphonohydrazides and gave the corresponding phosphonohydrazido oximes in excellent yields under mild conditions.
\end{abstract}

Keywords: Hydrazides; $\alpha$-ketooxime; marine fish toxin; phosphonohydrazido oximes; phenyl derivatives.

\section{Introduction}

The toxins associated with 'blooms', or explosive growth of certain marine dinoflagellates, have caused massive fish kills off the coasts of the United States, Canada, and Japan and have been the subject of intense studies in the last fifty years [1-2]. Although most of the 400 species of dinoflagellates are nontoxic, there are notable exception, i.e., the Gonyaulax species, which give rises 
to anaxitoxins and gonyautoxins[1-2] and Ptychodiscus brevis, which produces brevetoxins [3-4]. The dinoflagellate Ptychodiscus brevis has also been implicated in production of toxin red tide along the Gulf Coast of Florida [5-6]. Marine derived dinoflagellates have also become a rich source of structurally novel and pharmacologically active secondary metabolites [1]. Toxins produced by Ptychodiscus brevis is lipoidal in nature and their structures were established as $O, O$-dipropyl-(E)-2(1-methyl-2-oxopropylidene) phosphorohydrazidothiolate-(E)-oxime [7] and O,O-diphenylcyclooctylphosphoramidate (PB-1) on the basis of X-ray crystallography [8]. These marine toxins have attracted the attention of organic chemists due to their involvement in human intoxication and the resulting socio-economic impact of those incidents [9-11]. It is interesting to note that among naturally occurring fish toxins [7] the former is the only organophosphorus (OP) compound which contains thiophosphoryl moiety like other insecticides [12] and does not possess any good leaving group. Another unique feature of this compound is the presence of free oximino function, which is not found in any of the previously reported toxic OP compounds. One of the objectives of this study was to find out whether any of newly synthesized compounds can be used like the chemical nicolosamide, which is used for quick collection of fish from fresh water ponds. The non availability of $\mathrm{P}=\mathrm{O}$ analogues of naturally occurring fish toxins [7] from natural sources has prompted us to develop a general synthetic method for the preparation of $\mathrm{P}=\mathrm{O}$ and its analogues for complete toxicological and pharmacological studies. Moreover, $\mathrm{P}=\mathrm{O}$ compounds have been reported to be more toxic than the corresponding $\mathrm{P}=\mathrm{S}$ derivatives; for example paraoxon is more toxic than parathion [10, 12]. To explore its chemical and biological properties, we decided to develop a convenient method for the synthesis of $\mathrm{P}=\mathrm{O}$ analogues of naturally occurring fish toxins by doing structural modifications. In continuation of our ongoing research program on the synthesis of new derivatives of naturally occurring organophosphorus compounds [13], we have thus developed a method for synthesis of $N, N$-dialkylaminophenyl-2-(1alkyl/phenyl-2-oxopropylidene)phosphonohydrazido oximes and $O$-alkylphenyl-2-(1-alkyl/phenyl-2oxo-propylidene)phosphonohydrazido oximes 4 . To the best of our knowledge these compounds have not been reported in the literature.

\section{Result and Discussion}

Retrosynthetic analysis of the compounds suggested that these compounds could be synthesized from the corresponding phosphonohydrazides and the $\alpha$-ketooxime. In order to synthesize the target compounds, the intermediates 2, 3 were prepared by following literature procedures [14]. Various $N, N$ dialkylaminophenylphosphonohydrazides and $O$-alkylphenylphosphonohydrazides were condensed with substituted $\alpha$-ketooxime in the presence of activated silica in benzene at $80-90{ }^{\circ} \mathrm{C}$ to afford both $N, N$-dialkylaminophenyl-2-(1-alkyl/phenyl-2-oxopropylidene) phosphonohydrazido oximes and $O$ alkyl- phenyl-2-(1-alkyl/phenyl-2-oxopropylidene) phosphonohydrazido oximes 4 in excellent yield (Scheme 1, Table 1).

The reaction of $\mathrm{N}, \mathrm{N}$-dialkylamino- and $\mathrm{O}$-alkylphenylphosphonohydrazides with substituted diacetyl monoxime shown in Scheme 1 is apparently simple, but it requires a selective coupling of butane-2,3-dione monoxime (diacetyl monoxime) and corresponding phosphonohydrazides to obtain the desired compounds. Indeed, a variety of reagents are capable to converting a $\mathrm{C}=\mathrm{O}$ bond to a $\mathrm{C}=\mathrm{N}$ one. The traditional synthesis involves the condensation of a carbonyl group with $\mathrm{NH}_{2}-\mathrm{Y}(\mathrm{Y}=\mathrm{OH}, \mathrm{NH}$, $\mathrm{NHCONH}_{2}$, etc) through an addition elimination reaction. 
Scheme 1.

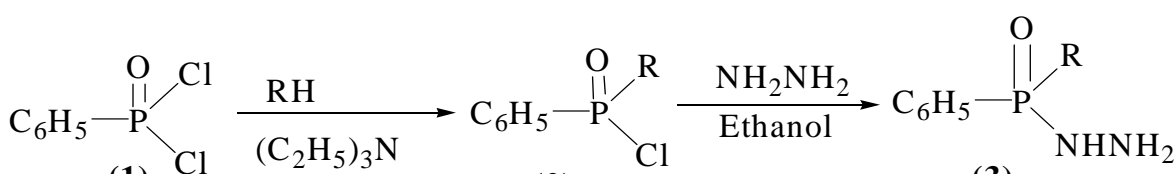

(1) $\mathrm{Cl}$ (2)

(3)<smiles>[R]C(=NNP([R])(=O)c1ccccc1)C(C)=NO</smiles>

Table-1 Physical data of the newly synthesized compounds $4^{\mathrm{a}}$.

\begin{tabular}{|c|c|c|c|c|c|c|}
\hline Entry & $\mathbf{R}$ & $\mathbf{R}^{1}$ & $\begin{array}{l}\text { Reaction } \\
\text { time (h) }\end{array}$ & ${ }^{31} \mathbf{P}-\mathrm{NMR}{ }^{\mathrm{b}}$ & $\begin{array}{l}\text { m. p. } \\
\left({ }^{0} \mathrm{C}\right)\end{array}$ & $\begin{array}{c}\text { Yield }^{c} \\
(\%)\end{array}$ \\
\hline $4 a$ & $\mathrm{~N}\left(\mathrm{C}_{2} \mathrm{H}_{5}\right)_{2}$ & $\mathrm{CH}_{3}$ & 3.0 & 22.55 & 154 & 81 \\
\hline $4 b$ & $\mathrm{~N}\left(\mathrm{C}_{3} \mathrm{H}_{7}\right)_{2}$ & $\mathrm{CH}_{3}$ & 3.2 & 23.09 & 158 & 85 \\
\hline $4 c$ & $\mathrm{~N}\left(\mathrm{C}_{3} \mathrm{H}_{7}\right)_{2}$ & $\mathrm{C}_{6} \mathrm{H}_{5}$ & 3.6 & 21.92 & 165 & 79 \\
\hline $4 d$ & $\mathrm{~N}\left(\mathrm{C}_{4} \mathrm{H}_{9}\right)_{2}$ & $\mathrm{CH}_{3}$ & 3.2 & 21.75 & 142 & 86 \\
\hline $4 e$ & $\mathrm{~N}\left(\mathrm{C}_{4} \mathrm{H}_{9}\right)_{2}$ & $\mathrm{C}_{6} \mathrm{H}_{5}$ & 4.2 & 21.63 & 178 & 93 \\
\hline 4f & $\mathrm{N}\left({ }^{\mathrm{i}} \mathrm{C}_{4} \mathrm{H}_{9}\right)_{2}$ & $\mathrm{CH}_{3}$ & 4.3 & 20.88 & 180 & 76 \\
\hline $4 g$ & $\mathrm{~N}\left({ }^{\mathrm{i}} \mathrm{C}_{4} \mathrm{H}_{9}\right)_{2}$ & $\mathrm{C}_{6} \mathrm{H}_{5}$ & 4.5 & 20.73 & 193 & 72 \\
\hline $4 h$ & $\mathrm{OC}_{3} \mathrm{H}_{7}$ & $\mathrm{CH}_{3}$ & 3.0 & 17.67 & 152 & 87 \\
\hline $4 i$ & $\mathrm{OC}_{3} \mathrm{H}_{7}$ & $\mathrm{C}_{6} \mathrm{H}_{5}$ & 3.1 & 17.27 & 155 & 74 \\
\hline $4 \mathbf{j}$ & $\mathrm{O}^{\mathrm{i}} \mathrm{C}_{3} \mathrm{H}_{7}$ & $\mathrm{CH}_{3}$ & 3.3 & 17.05 & 139 & 82 \\
\hline $4 \mathbf{k}$ & $\mathrm{O}^{\mathrm{i}} \mathrm{C}_{3} \mathrm{H}_{7}$ & $\mathrm{C}_{6} \mathrm{H}_{5}$ & 3.3 & 17.13 & 123 & 78 \\
\hline 41 & $\mathrm{OC}_{4} \mathrm{H}_{9}$ & $\mathrm{CH}_{3}$ & 3.1 & 17.41 & 154 & 73 \\
\hline $4 m$ & $\mathrm{O}^{\mathrm{i}} \mathrm{C}_{4} \mathrm{H}_{9}$ & $\mathrm{C}_{6} \mathrm{H}_{5}$ & 3.5 & 17.10 & 158 & 85 \\
\hline $4 n$ & $\mathrm{OC}_{5} \mathrm{H}_{11}$ & $\mathrm{CH}_{3}$ & 3.8 & 17.58 & 148 & 86 \\
\hline 40 & $\mathrm{OC}_{5} \mathrm{H}_{11}$ & $\mathrm{C}_{6} \mathrm{H}_{5}$ & 4.0 & 17.13 & 167 & 94 \\
\hline
\end{tabular}

a) All the reactions were performed in benzene under reflux conditions;

b) ${ }^{31} \mathrm{P}-\mathrm{NMR}$ data were recorded at $162 \mathrm{MHz}$ using either $\mathrm{CDCl}_{3}$ or $\mathrm{DMSO}_{6} \mathrm{~d}_{6}$ as a solvent; c) Isolated yields.

Initially, the more basic nitrogen of the hydrazides adds to the carbonyl moiety to furnish a tetrahedral intermediate, which transform the $\mathrm{C}=\mathrm{O}$ compounds to $\mathrm{C}=\mathrm{N}$ ones after elimination of water molecules. However, removal of water is a reversible process, thus it needs to be removed by 
azeotropic distillation or by using various dehydrating agents. However, the transformation of the $\mathrm{C}=\mathrm{O}$ double bond in the synthesis of $N, N$-dialkylaminophenyl-2-(1-alkyl/phenyl-2-oxopropylidene) phosphonohydrazido oximes and O-alkylphenyl-2-(1-alkyl/phenyl-2-oxopropylidene) phosphonohydrazido oximes is challenging due to the presence of reactive and free oxime $(\mathrm{N}=\mathrm{OH})$ and hydrazimino (-NH-N=) functionalities, which can undergo Beckmann rearrangements and/or cyclization reactions. This is probably the reason that no attempts have been made to synthesize compounds 4. Initially, the reaction of diethylaminophenylphosphonohydrazide with butane-2,3-dione monoxime was performed as a model reaction in the presence of various dehydrating reagents and by varying the reaction temperature. The efficiency of various dehydrating reagents such as $\mathrm{Al}_{2} \mathrm{O}_{3}$ (neutral, acidic, basic), $\mathrm{SiO}_{2}, \mathrm{ZnCl}_{2}, \mathrm{H}_{3} \mathrm{PO}_{4}, \mathrm{H}_{3} \mathrm{PO}_{3}, \mathrm{KF}-\mathrm{SiO}_{2}, \mathrm{POCl}_{3}, \mathrm{PTSA}, \mathrm{BiCl}_{3}, \mathrm{DCC}, \mathrm{MgSO}_{4}$, $\mathrm{V}_{2} \mathrm{O}_{5}-\mathrm{SiO}_{2}$ were studied at different molar ratios (10-120 mole \%). Amongst them anhydrous $\mathrm{SiO}_{2}$ gave the best result. In addition, to study the effect of solvent in above model reaction, various solvents like THF, dioxane, hexane, ethanol, diethyl ether, benzene, dichloromethane (DCM), chloroform and $\mathrm{CCl}_{4}$ were used and it was observed that benzene afforded the best results. During this study, we found that that isolated yield of $\mathbf{4}$ is affected by changing the solvent. Our studies revealed that the use of DCM, chloroform and $\mathrm{CCl}_{4}$ almost always gave more or less same yield of 4 (35-40\%) after longer reaction times and isolation of products from unreacted staring materials became difficult. Diethyl ether and hexane were not found to be an ideal choice of solvent due to the poor solubility of reactants in both the solvents, even under reflux conditions. The yields of 4 varied from 55-65\% when ethanol, dioxane or THF were used as solvents.

\section{Conclusions}

In conclusion, we have developed a more convenient and selective method for the synthesis of $N, N$-dialkylamino/O-alkyl phenyl-2-(1-alkyl/phenyl-2-oxopropylidene) phosphonohydrazido oximes 4. It was found that $\mathrm{SiO}_{2}$ promoted the reaction in high yields. The simplicity of the reaction conditions with short reaction times and without the use of column chromatography to obtain pure products in high yields should make this method attractive for organic chemists.

\section{Acknowledgements}

The authors thank Ms. Mamta Sharma and Avik Mazumder for NMR analysis.

\section{Experimental}

\section{General}

${ }^{1} \mathrm{H}-\mathrm{NMR},{ }^{31} \mathrm{P}-\mathrm{NMR}$ and ${ }^{13} \mathrm{C}-\mathrm{NMR}$ spectra $\left(\delta\right.$, ppm) were recorded in $\mathrm{CDCl}_{3} / \mathrm{DMSO}-\mathrm{d}_{6}$ solutions on a Bruker AVANCE 400 NMR spectrometer operating at 400, 162 and $100 \mathrm{MHz}$, respectively. Coupling constants $(J)$ are expressed in Hz. LCMS analysis (EI, 70V) were performed on a Hewlett-Packard HP 5971 instrument. IR spectra were recorded on a Bruker FT-IR spectrometer model Tensor 27 as $\mathrm{KBr}$ pellets. 
General procedure for the preparation of compounds 4a-o: N,N-diethylaminophenyl-2-(1-methyl-2oxopropylidene) phosphonohydrazido oxime (4a).

A mixture of butane-2,3-dione monoxime ( $1 \mathrm{~g}, 0.01 \mathrm{~mol})$ and activated silica gel (2 g) was placed in a two necked round bottom flask containing benzene $(20 \mathrm{~mL})$. N,N-diethylaminophenylphosphonohydrazide (2.27 $\mathrm{g}, 0.01 \mathrm{~mol})$ diluted in benzene $(20 \mathrm{~mL})$ was slowly added at room temperature with efficient stirring and the resulting mixture was refluxed for $3 \mathrm{~h}$. The reaction mixture was monitored by ${ }^{31} \mathrm{P}-\mathrm{NMR}$ until the $N, N$-diethylaminophenylphosphonohydrazide signal disappeared. The reaction mixture was filtered in a Buckner funnel and washed with benzene $(2 \times 10 \mathrm{~mL})$. The filtrate and washes were combined and solvent was removed by distillation. Finally, the desired crude product was triturated with dry ether gave a white crystalline powder which was crystallized from ethanol-ether (7:3); m. p. = 154; yield 81\%; IR ( $\left.\mathrm{cm}^{-1}\right)$ : 694 (P-C), 1089, $1152(\mathrm{P}-\mathrm{N}-\mathrm{C}), 1176(\mathrm{P}-\mathrm{N}-\mathrm{N})$, $1237(\mathrm{P}=\mathrm{O}), 1434(\mathrm{C}-\mathrm{N}), 1548(\mathrm{C}=\mathrm{C}), 1609(\mathrm{C}=\mathrm{N}), 2885(\mathrm{C}-\mathrm{H}), 2988\left(\mathrm{C}_{6} \mathrm{H}_{5}\right), 3317(\mathrm{OH}), 3361(\mathrm{NH})$; ${ }^{1} \mathrm{H}-\mathrm{NMR}: 1.03$ (t, $\left.J=8.02,6 \mathrm{H}, \mathrm{CH}_{3}\right), 1.88$ (s, 3H, $\left.\mathrm{CH}_{3}\right), 2.05$ (s, 3H, $\left.\mathrm{CH}_{3}\right), 3.05$ (m, $J=6.09,4 \mathrm{H}$, $\left.\mathrm{CH}_{2}\right), 6.60\left(\mathrm{~d}, J^{P-H}=30.26,1 \mathrm{H}, \mathrm{NH}\right) 7.4-7.79(\mathrm{~m}, J=6.79,5 \mathrm{H}, \mathrm{Ar}-\mathrm{H}), 8.01(\mathrm{~s}, 1 \mathrm{H}, \mathrm{OH}) ;{ }^{13} \mathrm{C}-\mathrm{NMR}$ : $9.33\left(\mathrm{CH}_{3}\right), 9.67\left(\mathrm{CH}_{3}\right), 23.16\left(\mathrm{CH}_{3}\right), 24.34\left(\mathrm{CH}_{3}\right), 39.05\left(\mathrm{CH}_{2}\right), 128-132(\mathrm{Ar}-\mathrm{C}), 145.39(\mathrm{C}=\mathrm{N}-\mathrm{NH})$, $156.16(\mathrm{C}=\mathrm{N}-\mathrm{OH}) ; \mathrm{MS}(\mathrm{m} / \mathrm{z}): 311\left(\mathrm{M}+\mathrm{H}^{+}\right), 333\left(\mathrm{M}+\mathrm{Na}^{+}\right) ; \mathrm{C}_{14} \mathrm{H}_{23} \mathrm{~N}_{4} \mathrm{O}_{2} \mathrm{P}$ Calcd. (Found) (\%): $\mathrm{C} 54.18$ (54.19), H 7.47 (7.50), N 18.05 (18.03).

N,N-dipropylaminophenyl-2-(1-methyl-2-oxopropylidene)phosphonohydrazido oxime (4b): IR ( $\left.\mathrm{cm}^{-1}\right)$ : 697 (P-C), 1070, 1150 (P-N-C), $1176(\mathrm{P}-\mathrm{N}-\mathrm{N}), 1232$ (P=O), 1427 (C-N),1544 (C=C), 1607 (C=N), $2850(\mathrm{C}-\mathrm{H}), 2948\left(\mathrm{C}_{6} \mathrm{H}_{5}\right), 3320(\mathrm{OH}), 3350(\mathrm{NH})$; ${ }^{1} \mathrm{H}-\mathrm{NMR}: 0.75$ (t, J=9.09, 6H, $\left.\mathrm{CH}_{3}\right), 1.45$ (m, $\left.J=8.73,4 \mathrm{H}, \mathrm{CH}_{2}\right), 1.88$ (s, 3H, $\left.\mathrm{CH}_{3}\right), 2.05$ (s, 3H, $\left.\mathrm{CH}_{3}\right), 2.85$ (m, J=8.56, 4H, $\mathrm{CH}_{2}$ ), 6.69 (d, $\left.J^{P-H}=27.28,1 \mathrm{H}, \mathrm{NH}\right), 7.32-7.79(\mathrm{~m}, J=7.42,5 \mathrm{H}, \mathrm{Ar}-\mathrm{H}), 9.5$ (s, $\left.1 \mathrm{H}, \mathrm{OH}\right) ;{ }^{13} \mathrm{C}-\mathrm{NMR}: 9.33\left(\mathrm{CH}_{3}\right), 21.82$ $\left(\mathrm{CH}_{2}\right), 23.16\left(\mathrm{CH}_{3}\right), 24.34\left(\mathrm{CH}_{3}\right), 47.08\left(\mathrm{CH}_{2}\right), 129-133(\mathrm{Ar}-\mathrm{C}), 145.40(\mathrm{C}=\mathrm{N}-\mathrm{NH}), 156.36(\mathrm{C}=\mathrm{N}-$ $\mathrm{OH}) ; \mathrm{MS}(\mathrm{m} / \mathrm{z}): 339\left(\mathrm{M}+\mathrm{H}^{+}\right), 361\left(\mathrm{M}+\mathrm{Na}^{+}\right) ; \mathrm{C}_{16} \mathrm{H}_{27} \mathrm{~N}_{4} \mathrm{O}_{2} \mathrm{P}$ Calcd. (Found) (\%): $\mathrm{C} 56.79$ (56.76), $\mathrm{H}$ 8.04 (8.08), N 16.56 (16.55).

N,N-dipropylaminophenyl-2-(1-phenyl-2-oxopropylidene)phosphonohydrazido oxime (4c): IR ( $\left.\mathrm{cm}^{-1}\right)$ : 694 (P-C), 996, 1110 (P-N-C), 1132 (P-N-N), $1193(\mathrm{P}=\mathrm{O}), 1450(\mathrm{C}-\mathrm{N}), 1554(\mathrm{C}=\mathrm{C}), 1647(\mathrm{C}=\mathrm{N})$, $2852(\mathrm{C}-\mathrm{H}), 2961\left(\mathrm{C}_{6} \mathrm{H}_{5}\right), 3147(\mathrm{OH}), 3290(\mathrm{NH}) ;{ }^{1} \mathrm{H}-\mathrm{NMR}: 0.75$ (t, $\left.J=9.23,6 \mathrm{H}, \mathrm{CH}_{3}\right), 1.50(\mathrm{~m}, J=$ 8.37, 4H, $\mathrm{CH}_{2}$ ), 2.05 (s, 3H, $\mathrm{CH}_{3}$ ), 2.85 (m, $J=8.37,4 \mathrm{H}, \mathrm{CH}_{2}$ ), 6.70 (d, $\left.J^{P-H}=30.77,1 \mathrm{H}, \mathrm{NH}\right), 7.15-7.81$ (m, J=7.69, 10H, Ar-H), 8.30 (s, 1H, OH); ${ }^{13} \mathrm{C}-\mathrm{NMR}: 9.33\left(\mathrm{CH}_{3}\right), 11.49\left(\mathrm{CH}_{3}\right), 21.74\left(\mathrm{CH}_{2}\right), 21.76$ $\left(\mathrm{CH}_{3}\right), 46.91\left(\mathrm{CH}_{2}\right), 128-132(\mathrm{Ar}-\mathrm{C}), 148.66(\mathrm{C}=\mathrm{N}-\mathrm{NH}), 157.16(\mathrm{C}=\mathrm{N}-\mathrm{OH}) ; \mathrm{MS}(\mathrm{m} / \mathrm{z}): 401\left(\mathrm{M}^{+} \mathrm{H}^{+}\right)$, $423\left(\mathrm{M}+\mathrm{Na}^{+}\right.$); $\mathrm{C}_{21} \mathrm{H}_{29} \mathrm{~N}_{4} \mathrm{O}_{2} \mathrm{P}$ Calcd. (Found) (\%): C 62.98 (62.95), H 7.30 (7.32), N 13.99 (13.97).

N,N-dibutylaminophenyl-2-(1-methyl-2-oxopropylidene)phosphonohydrazido oxime (4d): IR ( $\left.\mathrm{cm}^{-1}\right)$ : 703 (P-C), 1080, 1150 (P-N-C), 1173 (P-N-N), 1227 (P=O), 1437 (C-N), 1544 (C=C), $1609(\mathrm{C}=\mathrm{N})$, $2858(\mathrm{C}-\mathrm{H}), 2937\left(\mathrm{C}_{6} \mathrm{H}_{5}\right), 3307(\mathrm{OH}), 3355(\mathrm{NH}) ;{ }^{1} \mathrm{H}-\mathrm{NMR}: 0.83\left(\mathrm{t}, J=8.54,6 \mathrm{H}, \mathrm{CH}_{3}\right), 1.05(\mathrm{~m}, J=$ 
8.05, 4H, $\mathrm{CH}_{2}$ ), 1.35 (m, J=8.05, 4H, $\mathrm{CH}_{2}$ ), 1.78 (s, 3H, $\mathrm{CH}_{3}$ ), 1.95 (s, 3H, $\mathrm{CH}_{3}$ ), 3.05 (m, J=8.15, 4H, $\mathrm{CH}_{2}$ ), 6.85 (d, $\left.J^{P-H}=26.65,1 \mathrm{H}, \mathrm{NH}\right), 7.35-8.25$ (m, $\left.J=7.35,10 \mathrm{H}, \mathrm{Ar}-\mathrm{H}\right), 11.25$ (s, $\left.1 \mathrm{H}, \mathrm{OH}\right) ;{ }^{13} \mathrm{C}-\mathrm{NMR}$ : $9.37\left(\mathrm{CH}_{3}\right), 13.94\left(\mathrm{CH}_{2}\right), 20.05\left(\mathrm{CH}_{3}\right), 21.50\left(\mathrm{CH}_{3}\right), 30.71\left(\mathrm{CH}_{2}\right), 44.88\left(\mathrm{CH}_{2}\right), 128-132(\operatorname{Ar}-\mathrm{C})$, $146.49(\mathrm{C}=\mathrm{N}-\mathrm{NH}), 156.05(\mathrm{C}=\mathrm{N}-\mathrm{OH}) ; \mathrm{MS}(\mathrm{m} / \mathrm{z}): 367\left(\mathrm{M}+\mathrm{H}^{+}\right), 389\left(\mathrm{M}+\mathrm{Na}^{+}\right) ; \mathrm{C}_{18} \mathrm{H}_{31} \mathrm{~N}_{4} \mathrm{O}_{2} \mathrm{P}$ Calcd. (Found) (\%): C 59.00 (59.03), H 8.53 (8.50), N 15.29 (15.30).

N,N-dibutylaminophenyl-2-(1-phenyl-2-oxopropylidene)phosphonohydrazidooxime (4e): IR $\left(\mathrm{cm}^{-1}\right)$ : 694 (P-C), 1085, 1153 (P-N-C), 1166 (P-N-N), 1235 (P=O), 1454 (C-N), 1549 (C=C), $1609(\mathrm{C}=\mathrm{N})$, $2875(\mathrm{C}-\mathrm{H}), 2968\left(\mathrm{C}_{6} \mathrm{H}_{5}\right), 3307(\mathrm{OH}), 3352(\mathrm{NH})$; ${ }^{1} \mathrm{H}-\mathrm{NMR}: 0.85$ (t, J= 9.85, 6H, $\left.\mathrm{CH}_{3}\right), 1.15$ (m, $J=8.21,4 \mathrm{H}, \mathrm{CH}_{2}$ ), 1.55 (m, J=8.21, 4H, $\mathrm{CH}_{2}$ ), 1.95 (s, 3H, $\left.\mathrm{CH}_{3}\right), 2.85$ (m, J=8.35, 4H, $\mathrm{CH}_{2}$ ), 6.83 (d, $\left.J^{P-H}=27.60,1 \mathrm{H}, \mathrm{NH}\right), 7.25-7.85(\mathrm{~m}, J=7.84,10 \mathrm{H}, \mathrm{Ar}-\mathrm{H}), 10.25(\mathrm{~s}, 1 \mathrm{H}, \mathrm{OH}) ;{ }^{13} \mathrm{C}-\mathrm{NMR}: 9.30\left(\mathrm{CH}_{3}\right)$, $13.94\left(\mathrm{CH}_{2}\right), 21.50\left(\mathrm{CH}_{3}\right), 30.71\left(\mathrm{CH}_{2}\right), 44.88\left(\mathrm{CH}_{2}\right), 128-132(\mathrm{Ar}-\mathrm{C}), 146.49(\mathrm{C}=\mathrm{N}-\mathrm{NH}), 156.05$ $(\mathrm{C}=\mathrm{N}-\mathrm{OH}) ; \mathrm{MS}(\mathrm{m} / \mathrm{z}): 429\left(\mathrm{M}+\mathrm{H}^{+}\right) ; \mathrm{C}_{23} \mathrm{H}_{33} \mathrm{~N}_{4} \mathrm{O}_{2} \mathrm{P}$ Calcd. (Found) (\%): $\mathrm{C} 64.47$ (64.50), $\mathrm{H} 7.76$ (7.75), N 13.07 (13.05).

N,N-diisobutylaminophenyl-2-(1-methyl-2-oxopropylidene)phosphonohydrazido oxime (4f): IR ( $\left.\mathrm{cm}^{-1}\right)$ : 721 (P-C), 1076, 1152 (P-N-C), 1148 (P-N-N), $1240(\mathrm{P}=\mathrm{O}), 1452(\mathrm{C}-\mathrm{N}), 1557$ (C=C), $1609(\mathrm{C}=\mathrm{N})$, $2856(\mathrm{C}-\mathrm{H}), 2993\left(\mathrm{C}_{6} \mathrm{H}_{5}\right), 3298(\mathrm{OH}), 3345(\mathrm{NH})$; ${ }^{1} \mathrm{H}-\mathrm{NMR}: 0.70$ (d, J=8.23, 3H, $\left.\mathrm{CH}_{3}\right), 0.75$ (d, $J=8.23,3 \mathrm{H}, \mathrm{CH}_{3}$ ), 1.50 (m, J=8.25, 2H, CH), 1.78 (s, 3H, $\left.\mathrm{CH}_{3}\right), 1.95$ (s, 3H, $\mathrm{CH}_{3}$ ), 2.85 (m, J=8.25, $\left.4 \mathrm{H}, \mathrm{CH}_{2}\right), 8.10\left(\mathrm{~d}, J^{\mathrm{P}-\mathrm{H}}=29.05,1 \mathrm{H}, \mathrm{NH}\right), 7.35-7.85$ (m, 5H, J=7.49, Ar-H), 11.25 (s, $\left.1 \mathrm{H}, \mathrm{OH}\right) ;{ }^{13} \mathrm{C}-$ NMR: $9.45\left(\mathrm{CH}_{3}\right), 13.75\left(\mathrm{CH}_{3}\right), 15.45(\mathrm{CH}), 20.05\left(\mathrm{CH}_{3}\right), 1.50\left(\mathrm{CH}_{3}\right), 30.46\left(\mathrm{CH}_{2}\right), 128-132(\operatorname{Ar}-\mathrm{C})$, $146.49(\mathrm{C}=\mathrm{N}-\mathrm{NH}), 156.05(\mathrm{C}=\mathrm{N}-\mathrm{OH}) ; \mathrm{MS}(\mathrm{m} / \mathrm{z}): 367\left(\mathrm{M}+\mathrm{H}^{+}\right), 389\left(\mathrm{M}+\mathrm{Na}^{+}\right) ; \mathrm{C}_{18} \mathrm{H}_{31} \mathrm{~N}_{4} \mathrm{O}_{2} \mathrm{P}$ Calcd. (Found) (\%): C 59.00 (59.03), H 8.53 (8.50), N 15.29 (15.30).

$N, N$-diisobutylaminophenyl-2-(1-phenyl-2-oxopropylidene)phosphonohydrazido oxime (4g): IR ( $\left.\mathrm{cm}^{-1}\right)$ : 707 (P-C), 1095, 1148 (P-N-C), 1180 (P-N-N), $1230(\mathrm{P}=\mathrm{O}), 1425(\mathrm{C}-\mathrm{N}), 1557$ (C=C), $1606(\mathrm{C}=\mathrm{N})$, $2890(\mathrm{C}-\mathrm{H}), 2985\left(\mathrm{C}_{6} \mathrm{H}_{5}\right), 3311(\mathrm{OH}), 3356(\mathrm{NH}) ;{ }^{1} \mathrm{H}-\mathrm{NMR}: 0.70$ (d, 3H, J=8.77, $\left.\mathrm{CH}_{3}\right), 0.75$ (d, 3H, $\left.J=7.95, \mathrm{CH}_{3}\right), 1.50$ (m, J=7.95, 2H, CH), 1.78 (s, 3H, $\mathrm{CH}_{3}$ ), 1.95 (s, 3H, $\mathrm{CH}_{3}$ ), 2.85 (m, J=8.12, 4H, $\left.\mathrm{CH}_{2}\right), 6.65\left(\mathrm{~d}, J^{P-H}=28.69,1 \mathrm{H}, \mathrm{NH}\right), 7.35-7.85$ (m, $\left.J=7.98,10 \mathrm{H}, \mathrm{Ar}-\mathrm{H}\right), 11.25(\mathrm{~s}, 1 \mathrm{H}, \mathrm{OH}) ;{ }^{13} \mathrm{C}-\mathrm{NMR}$ : $9.68\left(\mathrm{CH}_{3}\right), 13.65\left(\mathrm{CH}_{3}\right), 20.47(\mathrm{CH}), 26.12\left(\mathrm{CH}_{3}\right), 52.55\left(\mathrm{CH}_{2}\right), 128-132(\mathrm{Ar}-\mathrm{C}), 148.56(\mathrm{C}=\mathrm{N}-\mathrm{NH})$, $157.60(\mathrm{C}=\mathrm{N}-\mathrm{OH}) ; \mathrm{MS}(\mathrm{m} / \mathrm{z}): 429\left(\mathrm{M}+\mathrm{H}^{+}\right), 451\left(\mathrm{M}+\mathrm{Na}^{+}\right) ; \mathrm{C}_{23} \mathrm{H}_{33} \mathrm{~N}_{4} \mathrm{O}_{2} \mathrm{P}$ Calcd. (Found) (\%): C 64.47, H 7.76, N 13.07; Found (\%): C 64.50, H7.75, N 13.05.

O-propylphenyl-2-(1-methyl-2-oxopropylidene)phosphonohydrazido oxime (4h): IR ( $\left.\mathrm{cm}^{-1}\right)$ : 705 (P-C), 1001, 1138 (P-O-C), 1193 (P=O), 1444 (C=C), 1587 (C=N), $2917(\mathrm{C}-\mathrm{H}), 2950\left(\mathrm{C}_{6} \mathrm{H}_{5}\right), 3191(\mathrm{OH})$, $3327(\mathrm{NH}) ;{ }^{1} \mathrm{H}-\mathrm{NMR}: 0.80$ (t, $\left.J=9.33,3 \mathrm{H}, \mathrm{CH}_{3}\right), 1.60$ (m, $\left.J=8.95,2 \mathrm{H}, \mathrm{CH}_{2}\right), 1.80\left(\mathrm{~s}, 3 \mathrm{H}, \mathrm{CH}_{3}\right), 1.92$ (s, $\left.3 \mathrm{H}, \mathrm{CH}_{3}\right), 3.90\left(\mathrm{~m}, J=9.36,2 \mathrm{H}, \mathrm{CH}_{2}\right), 8.35$ (d, $\left.J^{P-H}=26.67,1 \mathrm{H}, \mathrm{NH}\right), 7.35-7.85(\mathrm{~m}, J=7.96,5 \mathrm{H}$, 
Ar-H) 11.15 (s, 1H, OH); ${ }^{13} \mathrm{C}-\mathrm{NMR}: 9.30\left(\mathrm{CH}_{3}\right), 10.39\left(\mathrm{CH}_{2}\right), 23.79\left(\mathrm{CH}_{3}\right), \quad 23.85\left(\mathrm{CH}_{3}\right), 67.03$ $\left(\mathrm{CH}_{2}\right), 128-132$ (Ar-C), $147.15(\mathrm{C}=\mathrm{N}-\mathrm{NH}), 156.67(\mathrm{C}=\mathrm{N}-\mathrm{OH}) ; \mathrm{MS}(\mathrm{m} / \mathrm{z}): 298\left(\mathrm{M}^{+} \mathrm{H}^{+}\right), 320\left(\mathrm{M}^{+} \mathrm{Na}^{+}\right)$; $\mathrm{C}_{13} \mathrm{H}_{20} \mathrm{~N}_{3} \mathrm{O}_{3} \mathrm{P}$ Calcd. (Found) (\%): C 52.52 (52.55), H 6.78 (6.75), N 14.13 (14.10).

O-propylphenyl-2-(1-phenyl-2-oxopropylidene)phosphonohydrazido oxime (4i): IR ( $\left.\mathrm{cm}^{-1}\right)$ : 710 (P-C), 1011, 1129 (P-O-C), 1198 (P=O), 1445 (C=C), $1591(\mathrm{C}=\mathrm{N}), 2920(\mathrm{C}-\mathrm{H}), 2953\left(\mathrm{C}_{6} \mathrm{H}_{5}\right), 3198(\mathrm{OH})$, 3325 (NH); ${ }^{1} \mathrm{H}-\mathrm{NMR}: 0.80$ (t, J=8.53, 3H, $\mathrm{CH}_{3}$ ), 1.65 (m, J=8.37, 2H, $\mathrm{CH}_{2}$ ), 1.80 (s, 3H, $\mathrm{CH}_{3}$ ), 4.05 (m, $\left.J=9.21,2 \mathrm{H}, \mathrm{CH}_{2}\right), 6.85$ (d, $\left.J^{P-H}=28.87,1 \mathrm{H}, \mathrm{NH}\right), 7.35-7.85$ (m, J=7.86, 10H, Ar-H), 10.95 (s, 1H, $\mathrm{OH}) ;{ }^{13} \mathrm{C}-\mathrm{NMR}: 9.30\left(\mathrm{CH}_{3}\right), 10.39\left(\mathrm{CH}_{2}\right), 23.79\left(\mathrm{CH}_{3}\right), 66.97\left(\mathrm{CH}_{2}\right), 128-132(\mathrm{Ar}-\mathrm{C}), 147.15(\mathrm{C}=\mathrm{N}-$ $\mathrm{NH}), 156.67(\mathrm{C}=\mathrm{N}-\mathrm{OH}) ; \mathrm{MS}(\mathrm{m} / \mathrm{z}): 360\left(\mathrm{M}+\mathrm{H}^{+}\right), 382\left(\mathrm{M}+\mathrm{Na}^{+}\right) ; \mathrm{C}_{18} \mathrm{H}_{22} \mathrm{~N}_{3} \mathrm{O}_{3} \mathrm{P}$ Calcd. (Found) (\%): C 60.16 (60.15), H 6.17 (6.18), N 11.69 (11.67).

O-isopropylphenyl-2-(1-methyl-2-oxopropylidene)phosphonohydrazido oxime (4j): $\mathrm{IR}\left(\mathrm{cm}^{-1}\right): 715$ (P-C), 1012, 1125 (P-O-C), 1209 (P=O), 1456 (C=C), $1595(\mathrm{C}=\mathrm{N}), 2920(\mathrm{C}-\mathrm{H}), 2958\left(\mathrm{C}_{6} \mathrm{H}_{5}\right), 3197$ (OH), 3352 (NH); ${ }^{1} \mathrm{H}-\mathrm{NMR}: 1.25$ (d, J=5.65, 3H, $\mathrm{CH}_{3}$ ), 1.28 (d, J=6.67, 3H, $\mathrm{CH}_{3}$ ), 1.90 (s, 3H, $\mathrm{CH}_{3}$ ), 1.95 (s, 3H, $\mathrm{CH}_{3}$ ), 4.65 (m, J=7.41, 1H, CH), 6.85 (d, $\left.J^{P-H}=28.07,1 \mathrm{H}, \mathrm{NH}\right), 7.41-7.85$ (m, J= 8.5, 5H, Ar-H), 8.35 (s, 1H, OH); ${ }^{13} \mathrm{C}-\mathrm{NMR}: 9.28\left(\mathrm{CH}_{3}\right), 9.69\left(\mathrm{CH}_{3}\right), 23.86\left(\mathrm{CH}_{3}\right), 24.50\left(\mathrm{CH}_{3}\right), 70.88(\mathrm{CH})$,

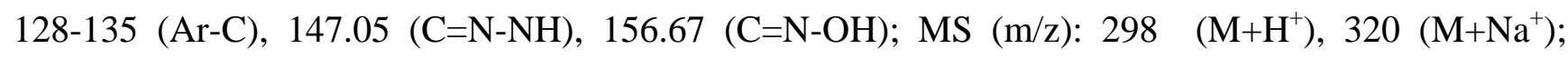
$\mathrm{C}_{13} \mathrm{H}_{20} \mathrm{~N}_{3} \mathrm{O}_{3}$ P Calcd. (Found) (\%): C 52.52 (52.55), H 6.78 (6.75), N 14.13 (14.10).

O-isopropylphenyl-2-(1-phenyl-2-oxopropylidene)phosphonohydrazido oxime (4k): IR ( $\left.\mathrm{cm}^{-1}\right): 705$ (P-C), 1015, 1135 (P-O-C), 1215 (P=O), 1453 (C=C), 1598 (C=N), $2922(\mathrm{C}-\mathrm{H}), 2948\left(\mathrm{C}_{6} \mathrm{H}_{5}\right), 3215$ (OH), 3319 (NH); ${ }^{1} \mathrm{H}-\mathrm{NMR}: 1.20$ (d, J=6.56, 3H, $\mathrm{CH}_{3}$ ), 1.30 (d, J=7.53, 3H, $\mathrm{CH}_{3}$ ), 2.05 (s, 3H, $\mathrm{CH}_{3}$ ), 4.65 (m, J=7.85, 1H, CH), 6.70 (d, $\left.J^{P-H}=26.15,1 \mathrm{H}, \mathrm{NH}\right), 7.41-7.85$ (m, J=7.98, 5H, Ar-H), 8.35 (s, 1H, $\mathrm{OH})$; ${ }^{13} \mathrm{C}-\mathrm{NMR}: 9.28\left(\mathrm{CH}_{3}\right), 9.69\left(\mathrm{CH}_{3}\right), 23.86\left(\mathrm{CH}_{3}\right), 70.88(\mathrm{CH}), 128-135(\mathrm{Ar}-\mathrm{C}), 147.05(\mathrm{C}=\mathrm{N}-$ $\mathrm{NH}), 156.67(\mathrm{C}=\mathrm{N}-\mathrm{OH}) ; \mathrm{MS}(\mathrm{m} / \mathrm{z}): 360\left(\mathrm{M}+\mathrm{H}^{+}\right), 382\left(\mathrm{M}+\mathrm{Na}^{+}\right) ; \mathrm{C}_{18} \mathrm{H}_{22} \mathrm{~N}_{3} \mathrm{O}_{3} \mathrm{P}$ Calcd. (Found) (\%): C 60.16 (60.17), H 6.17 (6.15), N 11.69 (11.68).

O-butylphenyl-2-(1-methyl-2-oxopropylidene)phosphonohydrazido oxime (4I): IR ( $\left.\mathrm{cm}^{-1}\right)$ : 705 (P-C), 1001, 1138 (P-O-C), 1193 (P=O), 1445 (C=C), 1587 (C=N), $2917(\mathrm{C}-\mathrm{H}), 2950\left(\mathrm{C}_{6} \mathrm{H}_{5}\right), 3191(\mathrm{OH})$, $3352(\mathrm{NH}) ;{ }^{1} \mathrm{H}-\mathrm{NMR}: 0.85$ (t, $\left.J=6.83,3 \mathrm{H}, \mathrm{CH}_{3}\right), 0.95$ (dd, $J=7.42,3 \mathrm{H}, \mathrm{CH}_{3}$ ), 1.20 (dd, $J=7.04,3 \mathrm{H}$, $\mathrm{CH}_{3}$ ), 1.40 (m, $J=7.23,2 \mathrm{H}, \mathrm{CH}_{2}$ ), 1.75 (m, $J=7.23,2 \mathrm{H}, \mathrm{CH}_{2}$ ), 2.10 (m, $\left.J=7.69,1 \mathrm{H}, \mathrm{CH}\right), 1.95$ (s, $3 \mathrm{H}, \mathrm{CH}_{3}$ ), 2.10 (s, 3H, $\mathrm{CH}_{3}$ ), 3.90 and 4.05 (m, $\left.J=7.43,2 \mathrm{H}, \mathrm{CH}_{2}\right), 6.55\left(\mathrm{~d}, J^{P-H}=20.35,1 \mathrm{H}, \mathrm{NH}\right), 7.58$ (s, $1 \mathrm{H}, \mathrm{OH}), 7.40-7.80$ (m, J= 7.95, 5H, Ar-H); ${ }^{13} \mathrm{C}-\mathrm{NMR}: 9.63\left(\mathrm{CH}_{3}\right), 15.87\left(\mathrm{CH}_{3}\right), 17.80\left(\mathrm{CH}_{2}\right)$, $19.20\left(\mathrm{CH}_{2}\right), 23.74\left(\mathrm{CH}_{3}\right), 24.46\left(\mathrm{CH}_{3}\right), 69.49\left(\mathrm{CH}_{2}\right), 128-135(\mathrm{Ar}-\mathrm{C}), 145.86(\mathrm{C}=\mathrm{N}-\mathrm{NH}), 157.44$ $(\mathrm{C}=\mathrm{N}-\mathrm{OH}) ; \mathrm{MS}(\mathrm{m} / \mathrm{z}): 312\left(\mathrm{M}+\mathrm{H}^{+}\right), 334\left(\mathrm{M}+\mathrm{Na}^{+}\right) ; \mathrm{C}_{14} \mathrm{H}_{22} \mathrm{~N}_{3} \mathrm{O}_{3} \mathrm{P}$ Calcd. (Found) (\%): $\mathrm{C} 54.01$ (54.02), H 7.12 (7.10), N 13.50 (13.53). 
O-isobutylphenyl-2-(1-phenyl-2-oxopropylidene)phosphonohydrazido oxime (4m): IR ( $\left.\mathrm{cm}^{-1}\right)$ : 712 (P-C), 1003, 1134 (P-O-C), 1197 (P=O), 1450 (C=C), 1585 (C=N), 2917 (C-H), $2956\left(\mathrm{C}_{6} \mathrm{H}_{5}\right), 3215$ $(\mathrm{OH}), 3347(\mathrm{NH}) ;{ }^{1} \mathrm{H}-\mathrm{NMR}: 0.75$ (d, $\left.J=8.56,3 \mathrm{H}, \mathrm{CH}_{3}\right), 0.80$ (d, $\left.J=8.25,3 \mathrm{H}, \mathrm{CH}_{3}\right), 1.80$ (s, $1 \mathrm{H}$, $\mathrm{CH}$ ), 2.05 (m, $J=8.36,1 \mathrm{H}, \mathrm{CH}$ ), 3.85 (m, $J=9.63,2 \mathrm{H}, \mathrm{CH}_{2}$ ), 6.70 (d, $\left.J^{P-H}=31.58,1 \mathrm{H}, \mathrm{NH}\right), 7.41-7.85$ (m, J=8.03, 10H, Ar-H), 8.50 (s, $1 \mathrm{H}, \mathrm{OH}) ;{ }^{13} \mathrm{C}-\mathrm{NMR}: 9.32\left(\mathrm{CH}_{3}\right), 9.80\left(\mathrm{CH}_{3}\right), 18.94(\mathrm{CH})$, 29.15( $\left.\mathrm{CH}_{3}\right), 71.26\left(\mathrm{CH}_{2}\right), 128-135$ (Ar-C), $147.37(\mathrm{C}=\mathrm{N}-\mathrm{NH}), 156.37(\mathrm{C}=\mathrm{N}-\mathrm{OH}) ; \mathrm{MS}(\mathrm{m} / \mathrm{z}): 374(\mathrm{M}+$ $\mathrm{H}^{+}$), $396\left(\mathrm{M}+\mathrm{Na}^{+}\right) ; \mathrm{C}_{19} \mathrm{H}_{24} \mathrm{~N}_{3} \mathrm{O}_{3} \mathrm{P}$ Calcd. (Found) (\%): C 61.12 (61.13), H 6.48 (6.47), N 11.25 (11.26).

O-pentylphenyl-2-(1-methyl-2-oxopropylidene)phosphonohydrazido oxime (4n): IR ( $\left.\mathrm{cm}^{-1}\right)$ : 711 (P-C), 997, 1130 (P-O-C), $193(\mathrm{P}=\mathrm{O}), 1437(\mathrm{C}=\mathrm{C}), 1601(\mathrm{C}=\mathrm{N}), 2852(\mathrm{C}-\mathrm{H}), 2943\left(\mathrm{C}_{6} \mathrm{H}_{5}\right), 3248(\mathrm{OH}), 3333$ (NH); ${ }^{1} \mathrm{H}-\mathrm{NMR}: 0.83$ (t, $\left.J=8.07,3 \mathrm{H}, \mathrm{CH}_{3}\right), 1.25$ (m, $\left.J=8.50,2 \mathrm{H}, \mathrm{CH}_{2}\right), 1.30\left(\mathrm{~m}, J=8.50,2 \mathrm{H}, \mathrm{CH}_{2}\right.$ ), 1.70 (m, $J=7.95,2 \mathrm{H}, \mathrm{CH}_{2}$ ), 1.85 (s, 3H, $\mathrm{CH}_{3}$ ), 1.97 (s, 3H, $\left.\mathrm{CH}_{3}\right), 4.10$ (m, $\left.J=8.15,2 \mathrm{H}, \mathrm{CH}_{2}\right), 6.75$ $\left(\mathrm{d}, J^{P-H}=24.67,1 \mathrm{H}, \mathrm{NH}\right), 7.35-7.90(\mathrm{~m}, J=8.35,5 \mathrm{H}, \mathrm{Ar}-\mathrm{H}), 7.7$ (s, 1H, OH); ${ }^{13} \mathrm{C}-\mathrm{NMR}: 9.27\left(\mathrm{CH}_{3}\right)$, $14.12\left(\mathrm{CH}_{2}\right), 22.38\left(\mathrm{CH}_{2}\right), 27.91\left(\mathrm{CH}_{2}\right), 30.10\left(\mathrm{CH}_{3}\right), 30.17\left(\mathrm{CH}_{3}\right), 65.47\left(\mathrm{CH}_{2}\right), 128-135(\operatorname{Ar}-\mathrm{C})$, $147.23(\mathrm{C}=\mathrm{N}-\mathrm{NH}), 156.77(\mathrm{C}=\mathrm{N}-\mathrm{OH}) ; \mathrm{MS}(\mathrm{m} / \mathrm{z}): 326\left(\mathrm{M}+\mathrm{H}^{+}\right), 348\left(\mathrm{M}+\mathrm{Na}^{+}\right) ; \mathrm{C}_{15} \mathrm{H}_{24} \mathrm{~N}_{3} \mathrm{O}_{3} \mathrm{P}$ Calcd. (Found) (\%): C 55.38 (55.37), H 7.44 (7.45), N 12.92 (12.90).

O-pentylphenyl-2-(1-phenyl-2-oxopropylidene)phosphonohydrazidooxime (4o): IR ( $\left.\mathrm{cm}^{-1}\right)$ : 695 (P-C), 987, 1125 (P-O-C), $1209(\mathrm{P}=\mathrm{O}), 1448(\mathrm{C}=\mathrm{C}), 1605(\mathrm{C}=\mathrm{N}), 2867(\mathrm{C}-\mathrm{H}), 2942\left(\mathrm{C}_{6} \mathrm{H}_{5}\right), 3228(\mathrm{OH})$, 3312 (NH); ${ }^{1} \mathrm{H}-\mathrm{NMR}: 0.90$ (t, $\left.J=8.45,3 \mathrm{H}, \mathrm{CH}_{3}\right), 1.35$ (m, J=8.40, 2H, $\mathrm{CH}_{2}$ ), 1.40 (m, J= 8.40, 2H, $\mathrm{CH}_{2}$ ), $1.72\left(\mathrm{~m}, J=8.50,2 \mathrm{H}, \mathrm{CH}_{2}\right), 2.10$ (s, 3H, $\left.\mathrm{CH}_{3}\right), 4.15$ (m, $\left.J=8.15,2 \mathrm{H}, \mathrm{CH}_{2}\right), 6.80\left(\mathrm{~d}, J^{P-H}=26.37\right.$, 1H, NH), 7.35-7.90 (m, J=8.35, 10H, Ar-H), 7.90 (s, $1 \mathrm{H}, \mathrm{OH}) ;{ }^{13} \mathrm{C}-\mathrm{NMR}: 9.60\left(\mathrm{CH}_{3}\right), 14.16\left(\mathrm{CH}_{2}\right)$, $22.41\left(\mathrm{CH}_{2}\right), 27.96\left(\mathrm{CH}_{2}\right), 30.20\left(\mathrm{CH}_{3}\right), 65.48\left(\mathrm{CH}_{2}\right), 128-132(\mathrm{Ar}-\mathrm{C}), 149.76(\mathrm{C}=\mathrm{N}-\mathrm{NH}), 157.33$ $(\mathrm{C}=\mathrm{N}-\mathrm{OH}) ; \mathrm{MS}(\mathrm{m} / \mathrm{z}): 388\left(\mathrm{M}+\mathrm{H}^{+}\right), 410\left(\mathrm{M}+\mathrm{Na}^{+}\right) ; \mathrm{C}_{20} \mathrm{H}_{26} \mathrm{~N}_{3} \mathrm{O}_{3} \mathrm{P}$ Calcd. (Found) (\%): C 62.00 (62.02), H 6.76 (6.77), N, 10.83 (10.85).

\section{References and Notes}

1. Alam, M.; Oshima, Y.; Shimizu, Y. About Gonyautoxins-1, -11, -111 and-1V. Tetrahedron Lett. 1982, 23, 321-322.

2. Shimizu, Y. In "Marine Natural Products"; Scheuer, P. J., Ed.; Academic Press: New York, 1978; Vol. 1, Chapter 1.

3. Lin, Y.Y.; Risk, M.; Ray, S.M.; Von Engen, D.; Clardy, J.; Golik, J.; James, J.C.; Nakanishi, K. Isolation and Structure of Brevetoxin B from the "Red Tide" Dinoflagellate Ptychodiscus brevis (Gymnodinium breve). J. Am. Chem. Soc. 1981, 103, 6773-6775.

4. James, J.C.; Golik, J.; Nakanishi, K. The Structure of Brevetoxin C. Tetrahedron Lett. 1982, 23, 2535-2538.

5. Martin, D. F.; Chatterjee, A. B. Some chemical and physical properties of two toxins from the red tide organism, Gymnodinium breve. U.S. Fish Wild. Serv. Fish. Bull. 1970, 68, 433-443. 
6. Sasner, J.J.; Ikawa, M.; Thurberg, F.; Alam, M. Physiological and chemical studies on Gymnodinium breve davis toxin. Toxicon 1972, 10, 163-172.

7. Alam, M.; Sanduja, R.; Hossain, M. B.; Helm, D. V. Gymnodinium breve Toxin. 1. Isolation and X-ray Structure of O,O-Dipropyl(E)-2-(1-methyl-2-oxoprolidene) phosphorohydrazidothiolate (E)Oxime from the Red Tide Dinoflagellate Gymnodinium breve. J. Am. Chem. Soc. 1982, 104, 5232-5234.

8. Dinovi, M.; Trainor, D. A.; Nakanishi, K. The structure of PB-1, an unusual toxin isolated from the red tide Dinoflagellate Ptychodiscus brevis. Tetrahedron Lett. 1983, 24, 855-858.

9. Kaushik, M. P.; Parashar, B. D.; Swamy, R.V. Synthesis of Gymnodinium breve Toxin. Ind. J. Chem. 1988, 27B, 1150-1151.

10. Husain, K.; Kaushik, M. P.; Gupta, A. K. Acute toxicity of synthetic Gymnodinium breve Toxin metabolite and its analogues in mice. Ecotox. Environ. Safety 1996, 35, 77-80.

11. (a) Koley, J.; Basak, A. K.; Das, M.; Sinha, S.; Dubey, S. N.; Majumder, P. K.; Gupta, A. K.; Das Gupta, S.; Koley, B. N. Effect of synthetic toxin of Ptychodiscus brevis on cardio-vascular and renal function. Ind. J. Physiol. Allied Sci. 1997, 51, 119-125; (b) Majumder, P. K.; Gupta, A. K.; Kaushik, M. P.; Deo Kumar; Dubey, S. N. Cardio-vascular effects of organophosphates toxin isolated from Ptychodiscus brevis. Biomed Environ. Sci. 1997, 10, 85-92; (c) Majumder, P. K.; Gupta, A. K.; Deo Kumar; Kaushik, M. P.; Dubey, S.N. Mechanism of cardiotoxicity induced by a marine toxin isolated from Ptychodiscus brevis. Ind. J. Expt. Bio. 1997, 35, 650-654; (d) Majumder, P. K.; Gupta, A. K.; Deo Kumar; Dubey, S.N. Calcium modulatory properties of O,Odiphenylcyclooctyl phosphoramidates from Ptychodiscus brevis in rat atria and smooth muscle. Ind. J. Physiol. Pharmacol. 1997, 41, 257-262.

12. Eto, M. “Organophosphorus Pesticides: Organic and Biological Chemistry”; CRC Press: Boca Raton, FL, USA, 1974; Chapter 5.

13. (a) Kumar, R.; Gupta, A. K.; Kaushik, M. P. A Novel and Efficient Synthesis of N,N-dialkylaminoisopropyl-2-(1-alkyl-2-oxopropylidene) phosphonohydrazido oximes and $O$-alkyl isopropyl2-(1-alkyl-2-oxopropylidene) phosphonohydrazido oximes - A potential marine fish toxin analogues. Part 1. Molecules 2007, 12, 1334-1340; (b) Gupta, A. K.; Dubey, D.K.; Sharma, M.; Kaushik, M. P. An efficient synthesis of the marine toxin Ptychodiscus brevis [PB-1] and its analogues. Org. Prep. Proced. Int. 2007, 39, 297-309; (c) Gupta, A. K.; Acharya, J.; Dubey, D.K.; Kaushik, M. P. Trichlorocyanuric Acid: An Efficient Reagent for One-Pot Synthesis of Ptychodiscus brevis (PB-1) Toxin and its Derivatives. J. Chem. Res. 2007, 29-33.

14. Audrieth, L. F.; Gher, R.; Smith, W.C. Nitrogen compounds of the phosphoric and phosphonic acids 1 . Hydrazides of phenylphosphoric and diphenylphosphoric acids. J. Org. Chem. 1955, 20, 1288-1290.

Sample Availability: Contact the authors.

(C) 2007 by MDPI (http://www.mdpi.org). Reproduction is permitted for noncommercial purposes. 